\title{
Spatial and Temporal Heterogeneity of Regional Myocardial Uptake in Patients Without Heart Disease Under Fasting Conditions on Repeated Whole-Body ${ }^{18}$ F-FDG PET/CT
}

\author{
Eugenio Inglese ${ }^{1}$, Lucia Leva ${ }^{1}$, Roberta Matheoud ${ }^{2}$, Gianmauro Sacchetti ${ }^{1}$, Chiara Secco ${ }^{2}$, Patrizia Gandolfo ${ }^{3}$, \\ Marco Brambilla ${ }^{2}$, and Gianmario Sambuceti ${ }^{3}$ \\ ${ }^{I}$ Nuclear Medicine Department, University of Eastern Piedmont, Azienda Ospedaliera Maggiore della Carità, Novara, Italy; \\ ${ }^{2}$ Medical Physics Department, Azienda Ospedaliera Maggiore della Carità, Novara, Italy; and ${ }^{3}$ Nuclear Medicine Department, \\ University of Genoa, Ospedale San Martino, Genova, Italy
}

Imaging of cardiac ${ }^{18} \mathrm{~F}-\mathrm{FDG}$ uptake is used in the diagnostic evaluation of residual viable myocardium. Although, originally, hibernating myocardium was identified by a mismatch between perfusion defect and relatively preserved ${ }^{18} \mathrm{~F}-\mathrm{FDG}$ uptake, at present several studies propose that ${ }^{18} \mathrm{~F}-\mathrm{FDG}$ distribution can also be used alone for this purpose. Nevertheless, even severe myocardial ${ }^{18} \mathrm{~F}$-FDG uptake defects are frequently observed in cancer patients without any cardiac disease. The aim of this study was to retrospectively analyze global and regional ${ }^{18} \mathrm{~F}$ FDG cardiac images of 49 consecutive cancer patients free of cardiac diseases who submitted to 3 PET scans under fasting conditions. Methods: Images were acquired with a high-resolution PET/CT scanner. Three-dimensional regions of interest were drawn on the fused PET/CT images to measure the maximal standardized uptake value of the left ventricular myocardium $\left(S_{U} V_{M y o}\right)$ as well as the average SUV of the left ventricular blood $\left(S U V_{L V}\right)$ and of the liver $\left(S_{U} V_{\text {Liver }}\right)$. Analysis of regional myocardial ${ }^{18} \mathrm{~F}-\mathrm{FDG}$ uptake was performed on a subsample of 26 patients by an automatic recognition of endocardial and epicardial borders and subdividing the left ventricle in 20 segments. Regional ${ }^{18} \mathrm{~F}-\mathrm{FDG}$ distribution was defined as the percentage of $\mathrm{SUV}_{\text {Myo }}$ in each region. Results: $\mathrm{SUV}_{\mathrm{Myo}}$ as well as $\mathrm{SUV}_{\mathrm{LV}}$ and $\mathrm{SUV}_{\mathrm{Liver}}$ did not change on average throughout the studies. This stability was not caused by a persistent pattern of myocardial ${ }^{18-F D G ~ d i s-~}$ tribution. Rather, it was associated with important variations in both directions over time. Regional ${ }^{18} \mathrm{~F}-\mathrm{FDG}$ distribution was largely heterogeneous in all 3 studies, with a variation coefficient in each patient of $18 \% \pm 7 \%, 18 \% \pm 5 \%$, and $17 \% \pm 5 \%$, respectively. An ${ }^{18} \mathrm{~F}-\mathrm{FDG}$ uptake of $<50 \%$ occurred in 78,102 , and 69 of 468 segments, although it disappeared in $55 \%$ of instances at subsequent examinations. Regional temporal variability was also marked: The absolute value of the difference in percent uptake was $10.1 \% \pm 7.3 \%$ from test 1 to test 2 , $8.0 \% \pm 7.0 \%$ from test 1 to test 3 , and $9.2 \% \pm 6.9 \%$ from test

Received Mar. 9, 2007; revision accepted Jul. 2, 2007.

For correspondence or reprints contact: Marco Brambilla, PhD, Medical Physics Department, A.O. Maggiore della Carità, C.so Mazzini 18, 28100 Novara, Italy.

E-mail: marco.brambilla@maggioreosp.novara.it

COPYRIGHT @ 2007 by the Society of Nuclear Medicine, Inc.
2 to test 3. Overall from one test to another, uptake increased or decreased by $>10 \%$ in 76 and in 116 of 468 segments, respectively. Conclusion: The large spatial and temporal heterogeneity of the myocardial metabolic pattern, in cancer patients free of any disease, suggests a word of caution on the use of ${ }^{18} \mathrm{~F}-\mathrm{FDG}$ alone as a diagnostic tool for myocardial viability.

Key Words: ${ }^{18}$ F-FDG; whole-body PET/CT; cardiac uptake

J Nucl Med 2007; 48:1662-1669

DOI: 10.2967/jnumed.107.041574

maging of cardiac ${ }^{18} \mathrm{~F}-\mathrm{FDG}$ uptake is used in the diagnostic evaluation of residual viable myocardium to identify candidates for revascularization among patients with postischemic left ventricular (LV) dysfunction. The rationale underlying this technique is that glucose consumption represents a basic function of myocardial cells that is preserved even in the presence of hypoperfusion and contractile dysfunction. Originally, the proposed diagnostic criterion for the diagnosis of hibernating myocardium was the mismatch between the presence of a perfusion defect and a relatively preserved ${ }^{18} \mathrm{~F}$-FDG uptake $(1,2)$. However, several other authors suggested that residual viability can be assessed either by measuring regional glucose consumption $(3,4)$ or by analyzing ${ }^{18} \mathrm{~F}$-FDG distribution throughout the LV walls $(5,6)$, with well-defined threshold values. This latter method is widely used and relies on the assumption that the metabolic pattern of the normal heart is homogeneous and is homogeneously influenced by environmental factors such as serum levels of metabolites and hormones or neuronal stimulation. Accordingly, heterogeneities in myocardial ${ }^{18} \mathrm{~F}-\mathrm{FDG}$ uptake reflect regional differences in the myocardial condition that, in turn, are considered diagnostic markers of disease.

This assumption is largely intuitive and is confirmed by the fact that the myocardial tissue is morphologically 
homogeneous, is characterized by a homogeneous perfusion, and displays a homogeneous response of contractility and blood flow to systemic stimulations. Nevertheless, it conflicts with the frequent observation of a largely heterogeneous ${ }^{18} \mathrm{~F}$-FDG uptake in the myocardium of healthy subjects (7) or patients undergoing whole-body PET for characterization of neoplastic disease and without any history of cardiac disorders (8).

Moreover, recent studies on the temporal variability in global cardiac ${ }^{18}$ F-FDG uptake in subjects undergoing repeated whole-body PET scans reported conflicting results $(9,10)$. Thus, no conclusive data are available so far to define whether the distribution of ${ }^{18} \mathrm{~F}-\mathrm{FDG}$ throughout myocardial walls changes over time or remains stable. Defining these different scenarios has the potential to provide an important insight into the regulation of regional myocardial metabolism as well as to allow a more accurate interpretation of myocardial ${ }^{18} \mathrm{~F}$-FDG uptake.

These studies led us to hypothesize that actually the spatial and temporal variability of myocardial ${ }^{18} \mathrm{~F}-\mathrm{FDG}$ uptake might result in a significant prevalence of transient severe defects on the PET images that could be mistaken for myocardial scar in the heart of subjects free of any cardiac disease. Accordingly, the purpose of this study was to quantitatively depict spatial and temporal variability of global and regional myocardial ${ }^{18} \mathrm{~F}$-FDG uptake in a series of noncardiac patients who submitted to whole-body PET for the characterization of a neoplastic disease.

\section{MATERIALS AND METHODS}

\section{Patient Population}

This is a retrospective analysis of ${ }^{18}$ F-FDG myocardial uptake in nondiabetic subjects undergoing serial PET scans for oncologic purpose. Patients with history of diabetes, chest pain, syncope, or dyspnea were excluded as were patients with abnormal findings on resting electrocardiography or echocardiography. Within our database, we identified 49 consecutive patients with 3 or more PET scans performed from September 2004 to May 2006 in the PET facility at the Nuclear Medicine Department at Novara. There were 31 male and 18 female patients (mean age \pm SD, $56 \pm 16 \mathrm{y}$; age range, 18-90 y). Primary indications for PET were Hodgkin's lymphoma $(n=11)$, non-Hodgkin's lymphoma $(n=11)$, lung cancer $(n=6)$, breast cancer $(n=5)$, search for primary tumor $(n=3)$, lower intestine cancer $(n=3)$, gastric cancer $(n=2)$, melanoma, uterus, anal, esophagus, thyroid, prostate, and urogenital tract cancer $(n=1)$, and retroperitoneal fibrosis $(n=1)$. The mean time interval between scan 1 and scan 2 in the same patient was $147 \mathrm{~d}$ (range, 40-323 d), whereas the mean time between scan 2 and scan 3 was of $137 \mathrm{~d}$ (range, 41-360 d). Table 1 shows the characteristics of the patients.

Prior radiotherapy (RT) occurred in 9 patients. In 4 of them (1 non-Hodgkin's lymphoma, 1 breast cancer, 1 lung cancer, 1 with metastasis of unknown origin), RT was performed within $1 \mathrm{y}$ of the first PET scan. In the remaining 5 patients ( 2 breast, 1 uterus, prostate, and thyroid cancer), RT took place $>2$ y before the first PET scan. Five additional patients underwent RT in the time elapsed between the first and second PET scans (2 lung, 1 esophagus, 1 Hodgkin's lymphoma, 1 non-Hodgkin's lymphoma) and 1 (thyroid cancer) had a second cycle of RT. Six additional patients underwent RT between the second and third PET scans (2 Hodgkin's lymphoma, 1 lung, 1 anal, 1 prostate, 1 breast cancer).

Prior chemotherapy occurred in 16 patients (4 lung, 3 breast, Hodgkin's lymphoma and non-Hodgkin's lymphoma, 1 uterus, urogenital tract, and lower intestine cancer). The status of each patient's chemotherapy treatment in the course of the study is depicted in Figure 1.

In a subgroup of 26 patients ( 15 male, 11 female) with a mean age $\pm \mathrm{SD}$ of $58 \pm 15 \mathrm{y}$ (range, 27-79 y), an automatic semiquantitative regional analysis of cardiac uptake was feasible and was performed for the 3 scans. Primary indications for PET were Hodgkin's lymphoma $(n=8)$, breast cancer $(n=4)$, lung cancer $(n=4)$, non-Hodgkin's lymphoma $(n=3)$, search for primary tumor $(n=$ $3)$, melanoma $(n=1)$, gastric cancer $(n=1)$, uterus cancer $(n=1)$, and anal cancer $(n=1)$. The mean time interval between scan 1 and scan 2 in the same patient was $152 \mathrm{~d}$ (range, 75-300 d), whereas the mean time between scan 2 and scan 3 was $144 \mathrm{~d}$ (range, 41-316 d).

TABLE 1

Characteristics of Study Population at 3 Study Times

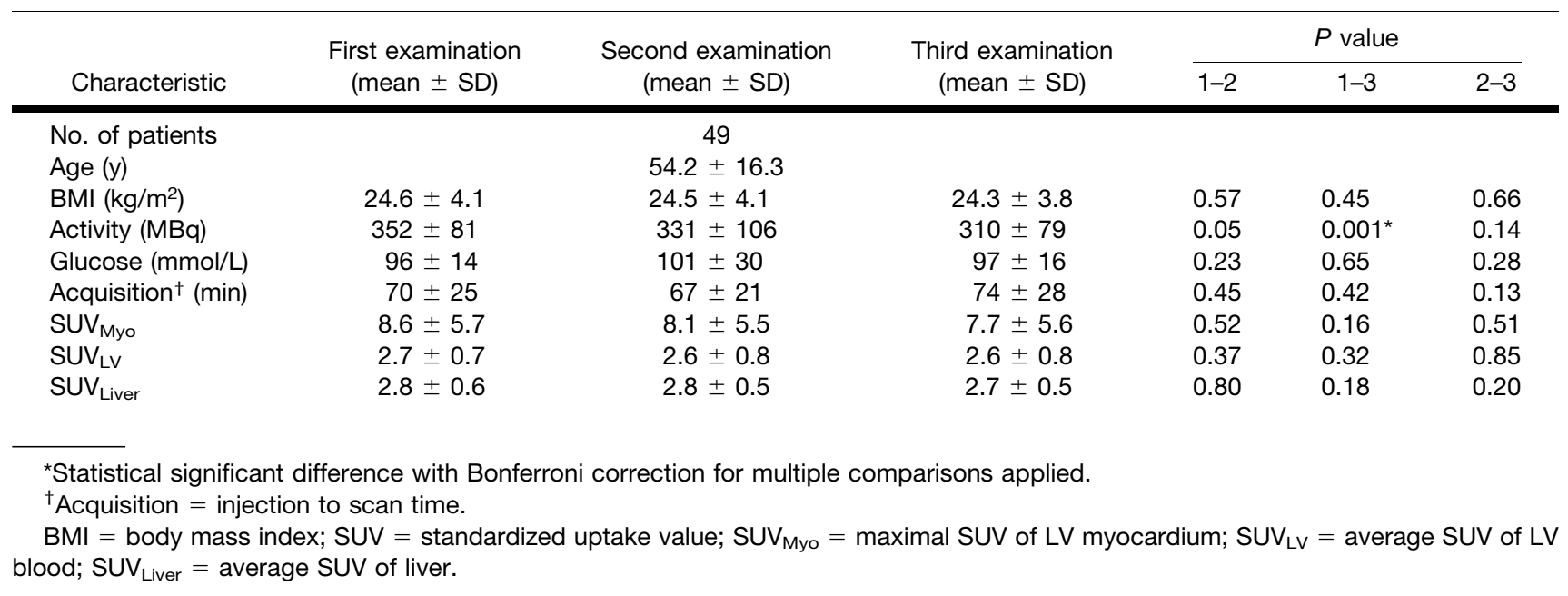




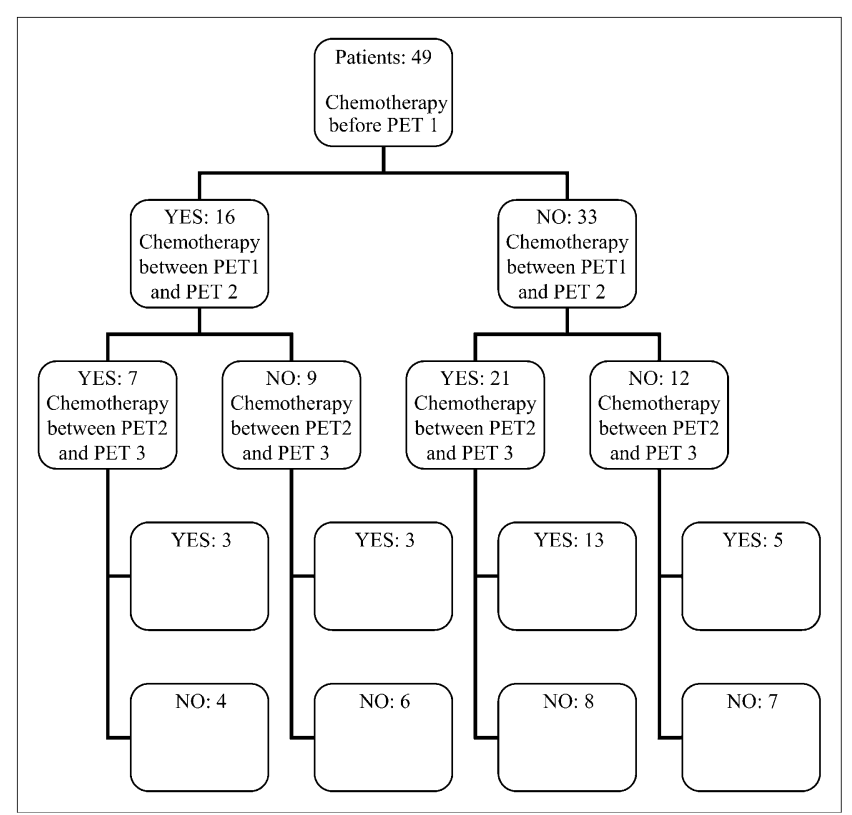

FIGURE 1. Status of administered chemotherapy to patient population in course of the study.

\section{PET Protocol}

Images were acquired with a Biograph 16 HI-REZ PET/CT scanner (Siemens Medical Solutions, Inc.), equipped with Pico-3D digital electronics. The PET component is a high-resolution scanner with a spatial resolution of $4.7 \mathrm{~mm}$ and has no septa, thus allowing 3-dimensional-only acquisitions. The CT portion of the scanner is the Somatom Sensation 16-slice CT. Together with the PET system, the CT scanner is used both for attenuation correction of PET data and for localization of ${ }^{18}$ F-FDG uptake in PET images.

All patients were advised to fast for at least $6 \mathrm{~h}$ before the integrated PET/CT examination. After injection of about $5 \mathrm{MBq}$ of ${ }^{18} \mathrm{~F}-\mathrm{FDG}$ per kilogram of body weight, patients rested for a period of about $60 \mathrm{~min}$ in a comfortable chair. Emission images ranging from the proximal femur and the base of the skull were acquired for 2-3 min per bed position. Acquired images were reconstructed using the attenuation weighted-OSEM (orderedsubset expectation maximization) iterative reconstruction, with 2 iterations, 8 subsets. Fourier rebinning was used to reduce the 3D dataset to a 2-dimensional equivalent dataset, and a 4-mm full width at half maximum gaussian filter was applied to the image after reconstruction along the axial and transaxial directions. The data were reconstructed over a $128 \times 128$ matrix with $5.25-\mathrm{mm}$ pixel size and 2-mm slice thickness. Processed images were displayed in coronal, transverse, and sagittal planes.

\section{Image Analysis}

Three-dimensional regions of interest (ROIs) were drawn on the fused PET/CT images to measure the maximal standardized uptake value of the $\mathrm{LV}$ myocardium $\left(\mathrm{SUV}_{\mathrm{Myo}}\right)$ as well as the average SUV of the LV blood $\left(\mathrm{SUV}_{\mathrm{LV}}\right)$ and of the liver $\left(\mathrm{SUV}_{\text {Liver }}\right)$. The SUV was computed according to the classical formulation as: $\mathrm{SUV}=$ (peak $\mathrm{kBq} / \mathrm{mL}$ in ROI)/injected activity/g body weight). For the determination of the $\mathrm{SUV}_{\mathrm{LV}}$, an ROI of $3.0 \mathrm{~cm}^{3}$ was placed over the $\mathrm{LV}$ chamber near the mitral valve plane. For the determination of $\mathrm{SUV}_{\text {Liver, }}$, an ROI of $6.6 \mathrm{~cm}^{3}$ was placed over the IV-V liver segment.

In a subgroup of 26 patients, the semiquantitative analysis of regional myocardial ${ }^{18} \mathrm{~F}$-FDG uptake was performed using a commercially available software package (Cedars QPS; Cedars-Sinai Medical Center, Los Angeles, CA) (11), which allows an operatorindependent recognition of endocardial and epicardial borders. In the present study, the software was used to automatically generate polar maps that were subdivided into 20 segments according to the commercially available QPS protocol (Fig. 2). In each study, the 2 apical segments were excluded from the analysis to avoid variability related to inaccuracies in the definition of apical thickness. Regional myocardial ${ }^{18} \mathrm{~F}$-FDG uptake was expressed as a percentage value of the $\mathrm{SUV}_{\mathrm{Myo}}$. Necrotic pattern was defined as a relative tracer uptake of $<50 \%$ of maximal myocardial uptake.

\section{Statistical Analysis}

Data are shown as mean \pm 1 SD. Pearson correlation coefficients were calculated among ${ }^{18}$ F-FDG uptake indices and clinical parameters.

The temporal reproducibility $(\mathrm{R})$ of myocardial, $\mathrm{LV}$, and liver uptake was estimated by the intraclass correlation coefficient
FIGURE 2. Serial myocardial ${ }^{18} \mathrm{FDG}$ PET results of 58-y-old male patient with non-Hodgkin's lymphoma who submitted 3 times to whole-body PET/CT. Shortaxis, horizontal long-axis, and vertical long-axis slices are displayed together with polar maps of the semiquantitative QPS software. Overlays of 20 myocardial segments, coronary artery territories, and myocardial walls are represented on top, middle, and bottom 2-dimensional polar maps, respectively. Patient was asymptomatic and completely free of any sign of cardiovascular diseases during observation period. After ${ }^{18} \mathrm{~F}-\mathrm{FDG}$ injection in fasting conditions, with blood glucose levels never exceeding $100 \mathrm{mg} / \mathrm{dL}$, a marked variability of regional myocardial uptake over time is evident.
Study 1

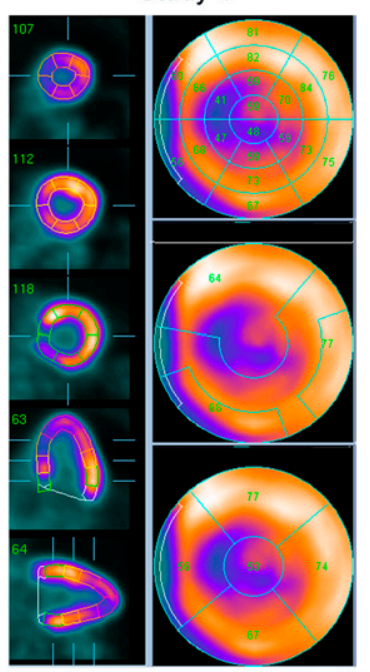

Study 2

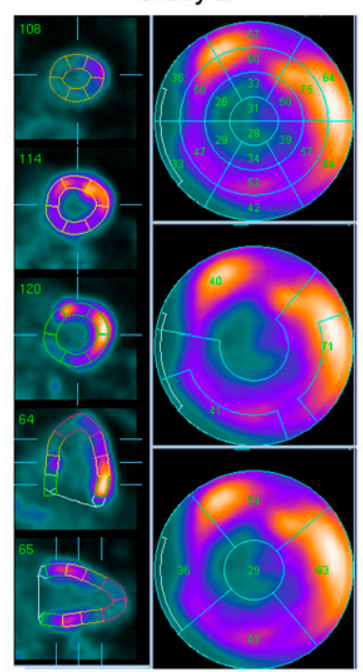

Study 3

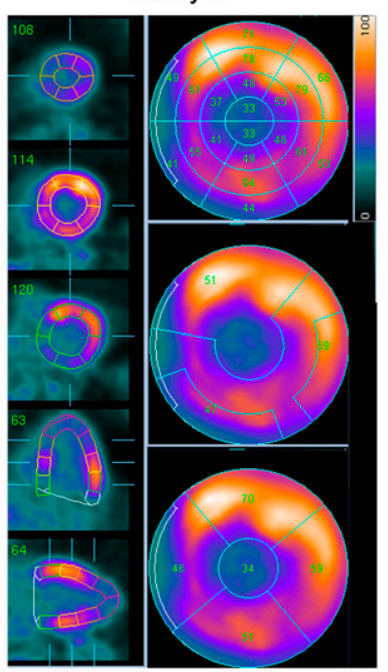


(ICC) using variance component estimation (12). Briefly, R was determined as the variance between subjects $\left(\sigma_{\text {sub }}^{2}\right)$ divided by the sum of error variance $\left(\sigma_{\text {err }}^{2}\right)$, observation variance $\left(\sigma_{\text {obs }}^{2}\right)$, and the variance between subjects:

$$
R=\frac{\sigma_{s u b}^{2}}{\sigma_{s u b}^{2}+\sigma_{o b s}^{2}+\sigma_{e r r}^{2}} .
$$

Thus, $\mathrm{R}$ is expressed as a number between 0 and 1 , with 0 indicating no $\mathrm{R}$ and 1 indicating a perfect $\mathrm{R}$. A value of 0.75 is a fairly minimum requirement for a useful measure. For instance, an $\mathrm{R}$ of 0.8 and 0.95 will result in a $20 \%$ and $2.2 \%$ chance of reversal of the order of 2 scores separated by an interquartile distance in repeated testing (13). Temporal heterogeneity of regional uptake was also expressed by the absolute value of the difference in regional percent myocardial uptake among tests 1, 2, and 3 . Spatial heterogeneity of global myocardial ${ }^{18} \mathrm{~F}-\mathrm{FDG}$ uptake was described by percent coefficient of variation $(\mathrm{SD} /$ mean \%) in each patient and in each study. Myocardial segments provided by quantitative polar map analysis were grouped for regional analysis according to coronary supply vessel (left anterior descending [LAD], left circumflex [LCX], right coronary artery [RCA]), myocardial walls (anterior, lateral, posterior, septum), and relative position of territories with respect to heart base (proximal, medial, distal) as shown in Figure 2. One-way ANOVA was used to estimate the effect of these groupings on regional ${ }^{18} \mathrm{~F}$-FDG uptake. Post hoc comparisons were performed using $t$ tests corrected for the number of comparisons by the Bonferroni method. Probability $(P)$ values of $<0.05$ were considered statistically significant. All data analysis was performed with Statistica 6.0 software (Statsoft).

\section{RESULTS}

No patient had any cardiac event during the observation period. Table 1 shows clinical variables and documents that no systematic changes occurred in average body mass index (BMI), serum glucose level, and injection to scan time at the 3 studies. Only a slight diminishing trend was observed for activity administered between study 1 and study 3 .

As reported in Table 2, intervening RT centered on the chest did not affect the metabolic pattern of the myocardium or the liver nor did it modify the heterogeneity in ${ }^{18} \mathrm{~F}-\mathrm{FDG}$ distribution throughout the LV walls. Similarly, as shown in Table 3, intervening chemotherapy did not modify the metabolic pattern or its heterogeneous distribution within the LV myocardium.

SUV $_{\text {Myo }}$ did not change on average throughout the studies; similarly, no systematic changes occurred in $\mathrm{SUV}_{\mathrm{LV}}$ or $\mathrm{SUV}_{\text {Liver, }}$ as reported in Table 1. No significant correlations were found between $\mathrm{SUV}_{\mathrm{Myo}}$ and age $(r=-0.06)$, BMI $(r=-0.11)$, blood glucose level $(r=-0.05)$, and injected activity $(r=-0.17)$. $\mathrm{SUV}_{\mathrm{Myo}}$ is not correlated with $\operatorname{SUV}_{\text {Liver }}(r=-0.18 ; P=$ not significant), whereas, as expected, it is significantly correlated with $\operatorname{SUV}_{\mathrm{LV}}(r=$ $0.48 ; P<0.05)$.

\section{Temporal Heterogeneity of Global and Regional Myocardial ${ }^{18} \mathrm{~F}-\mathrm{FDG}$ Uptake}

Global myocardial uptake of ${ }^{18} \mathrm{~F}-\mathrm{FDG}$ displayed a significant variability over time. There was a poor temporal reproducibility of $\mathrm{SUV}_{\mathrm{Myo}}$ on serial PET scans $(\mathrm{ICC}=$ $0.61)$. A still lower reproducibility was observed for $\mathrm{SUV}_{\mathrm{LV}}$ $(\mathrm{ICC}=0.42)$ and for $\mathrm{SUV}_{\text {Liver }}(\mathrm{ICC}=0.30)$. This demonstrate that, although average SUVs did not change from one study to another, this stability was not caused by a persistent pattern of myocardial ${ }^{18} \mathrm{~F}-\mathrm{FDG}$ distribution. Rather it was associated with important variations in both directions over time. The temporal reproducibility of regional uptake on repeated imaging was also poor (ICC $=$ 0.44 ). Indeed, the absolute value of the difference in percent uptake amount to $10.1 \% \pm 7.3 \%$ from test 1 to test $2,8.0 \% \pm 7.0 \%$ from test 1 to test 3 , and $9.2 \% \pm 6.9 \%$ from test 2 to test 3 . Overall, 76 of 468 segments (16.2\%) showed an increase in uptake of $>10 \%$, whereas 116 (24.7\%) showed a worsening of $>10 \%$ between test 1 and test 2 . When analyzing their behavior on the third scan, we found that 41 of 76 segments with an initial increase diminished their uptake of $>10 \%$, whereas 51 of 116 segments with an initial decrease improved their uptake of $>10 \%$. Moreover, the net difference between percent uptake in 2 different tests displayed a significant correlation with the percent uptake value at the first test,

TABLE 2

Effect of RT on PET Descriptor of Myocardial Metabolic Pattern

\begin{tabular}{|c|c|c|c|}
\hline \multicolumn{4}{|c|}{ RT between PET1 and PET2 $(n=5)$} \\
\hline Parameter & Findings at PET1 & Findings at PET2 & $P$ value \\
\hline SUV $_{\text {Myo }}$ & $9.6 \pm 4.3$ & $11.0 \pm 2.6$ & 0.50 \\
\hline SUV $_{\text {LV }}$ & $3.1 \pm 0.4$ & $2.9 \pm 0.5$ & 0.39 \\
\hline SUV $_{\text {Liver }}$ & $3.1 \pm 0.3$ & $3.1 \pm 0.7$ & 0.97 \\
\hline Coefficient of variation (\%) & $20.8 \pm 4.8(n=3)$ & $21.2 \pm 2.6$ & 0.77 \\
\hline \multicolumn{4}{|c|}{ RT between PET2 and PET3 $(n=4)$} \\
\hline Parameter & Findings at PET2 & Findings at PET3 & $P$ value \\
\hline SUV $_{\text {Myo }}$ & $11.6 \pm 7.4$ & $14.4 \pm 7.3$ & 0.45 \\
\hline SUV $_{\mathrm{LV}}$ & $2.9 \pm 0.7$ & $3.2 \pm 0.6$ & 0.45 \\
\hline SUV $_{\text {Liver }}$ & $2.7 \pm 0.5$ & $2.5 \pm 0.9$ & 0.50 \\
\hline Coefficient of variation (\%) & $14.9 \pm 2.9(n=3)$ & $14.7 \pm 3.5$ & 0.96 \\
\hline
\end{tabular}


TABLE 3

Effect of Chemotherapy on PET Descriptor of Myocardial Metabolic Pattern

\begin{tabular}{|c|c|c|c|}
\hline \multicolumn{4}{|c|}{ Chemotherapy between PET1 and PET2 $(n=28)$} \\
\hline Parameter & Findings at PET1 & Findings at PET2 & $P$ value \\
\hline $\begin{array}{l}\text { SUV }_{\text {Myo }} \\
\text { SUV } \\
\text { LV } \\
\text { SUV } \\
\text { Coeffici } \\
\text { Coefient of variation (\%) }\end{array}$ & $\begin{aligned} 7.7 & \pm 4.5 \\
2.5 & \pm 0.7 \\
2.7 & \pm 0.6 \\
19.0 & \pm 8.8(n=13)\end{aligned}$ & $\begin{array}{r}7.1 \pm 4.3 \\
2.5 \pm 0.8 \\
3.1 \pm 0.7 \\
18.1 \pm 4.4\end{array}$ & $\begin{array}{l}0.49 \\
0.80 \\
0.69 \\
0.60\end{array}$ \\
\hline \multicolumn{4}{|c|}{ Chemotherapy between PET2 and PET3 $(n=24)$} \\
\hline Parameter & Findings at PET2 & Findings at PET3 & $P$ value \\
\hline $\begin{array}{l}\text { SUV }_{\text {Myo }} \\
\text { SUV } \\
\text { LV } \\
\text { SUV } \\
\text { Coefiver } \\
\text { Coefient of variation (\%) }\end{array}$ & $\begin{aligned} 7.5 & \pm 5.3 \\
2.4 & \pm 0.7 \\
2.7 & \pm 0.5 \\
17.8 & \pm 6.0(n=11)\end{aligned}$ & $\begin{array}{r}7.2 \pm 5.3 \\
2.5 \pm 0.9 \\
2.6 \pm 0.7 \\
17.6 \pm 5.9\end{array}$ & $\begin{array}{l}0.81 \\
0.74 \\
0.36 \\
0.80\end{array}$ \\
\hline
\end{tabular}

indicating that the lower the uptake at the first scan the larger the increase in uptake from this study to the next one (Fig. 3).

\section{Spatial Heterogeneity of Regional Myocardial ${ }^{18}$ F-FDG Uptake}

Regional myocardial ${ }^{18} \mathrm{~F}$-FDG uptake was largely heterogeneous in all 3 studies. The average variation coefficient in each patient was $18 \% \pm 7 \%, 18 \% \pm 5 \%$, and $17 \% \pm 5 \%$ for the 3 studies, respectively. This index of heterogeneity was not predicted by any clinical variable or by the $\mathrm{SUV}_{\text {Myo. }}$ Necrotic pattern occurred in 78, 102, and 69 of 468 segments, respectively $(17 \%, 22 \%$ and $15 \%)$ and occurred in $\geq 2$ contiguous segments in 13,11 , and 10 patients at scans 1, 2, and 3, respectively (Fig. 4). Among them, a significant fraction ( $32 \%$ between first and second study and $55 \%$ between second and third study) showed a significant reversibility (uptake $\geq 50 \%$ ) at subsequent examinations.

When myocardium was subdivided according to coronary branch territories, mean uptake was higher in LCX $(67.5 \% \pm 11.4 \%)$ than that in RCA territories $(62.8 \% \pm$ $11.8 \% ; P=0.0011)$. This latter uptake was higher than that in LAD territories $(58.9 \% \pm 11.7 \% ; P=0.01)$. Similarly, lateral wall uptake $(67.5 \% \pm 11.4 \%)$ was significantly higher than that in the anterior wall $(60.0 \% \pm 11.6 \% ; P<$ $\left.10^{-4}\right)$ and septum $\left(59.7 \% \pm 12.3 \% ; P<10^{-6}\right) .{ }^{18} \mathrm{~F}-\mathrm{FDG}$ uptake in the inferior wall was an intermediate value $(64.2 \% \pm 10.6 \%)$. Finally, basal-apical gradient analysis showed that uptake in middle territories $(67.7 \% \pm 12.2 \%)$ was significantly higher than that in both proximal $(61.4 \% \pm$ $10.5 \% ; P<0.001)$ or distal ones $(60.1 \% \pm 12.3 \% ; P<$ $0.001)$.

\section{DISCUSSION}

The present study shows that ${ }^{18}$ F-FDG imaging indicates a large spatial and temporal heterogeneity of myocardial metabolic pattern under fasting conditions in cancer patients free of any clinically detectable heart disease. Thus, these data indicate that metabolic propensity to glucose use largely varies across different regions at the same time and at different times in the same region even in the presence of a relatively stable average glucose consumption in the whole heart. This observation has at least 2 major implications: first, on a pathophysiologic basis, it indicates that myocardial metabolism is influenced not only by systemic variables (such as serum concentrations of metabolite and related hormones) but also by largely unknown variables that are specific to each myocardial cell and to each time point, even in patients without cardiac disease; second, on the clinical basis, it suggests a word of caution on the use of ${ }^{18}$ F-FDG imaging alone as a diagnostic tool for the identification of myocardial viability.

\section{Spatial Heterogeneity of Regional Myocardial ${ }^{18}$ F-FDG Uptake}

Consistent with previous observations $(7,14,15)$, myocardial ${ }^{18}$ F-FDG uptake was distributed heterogeneously throughout the LV myocardium, with a relatively higher uptake in the lateral and posterior walls than in the anterior wall and septum. On the contrary, we did not observe the consistent basal-apical gradient in ${ }^{18} \mathrm{~F}-\mathrm{FDG}$ concentration that has been reported by Fukuchi et al. (8). Actually, RT might have induced regional myocardial damage, thus explaining the heterogeneous myocardial uptake at least in the subset of patients who underwent this treatment. However, this hypothesis is not confirmed by the data as serial interrogations of myocardial ${ }^{18}$ F-FDG distribution documented quite similar patterns before and after RT.

It is well known that physiologic and metabolic status can markedly affect heart metabolism and, thus, cardiac uptake of ${ }^{18}$ F-FDG. Accordingly, image quality might vary considerably, even in the same subject at different time points. However, we reduced this possible limitation a priori by selecting those patients in whom cardiac ${ }^{18} \mathrm{~F}-\mathrm{FDG}$ uptake was clearly visible at all study points. In agreement with this inclusion criterion, the quality of analyzed images remained relatively stable in this subset of patients. This finding is confirmed by the fact that, on average, no significant changes occurred in the heart-to-blood concentration ratio as well as in maximal myocardial SUV. Moreover, 


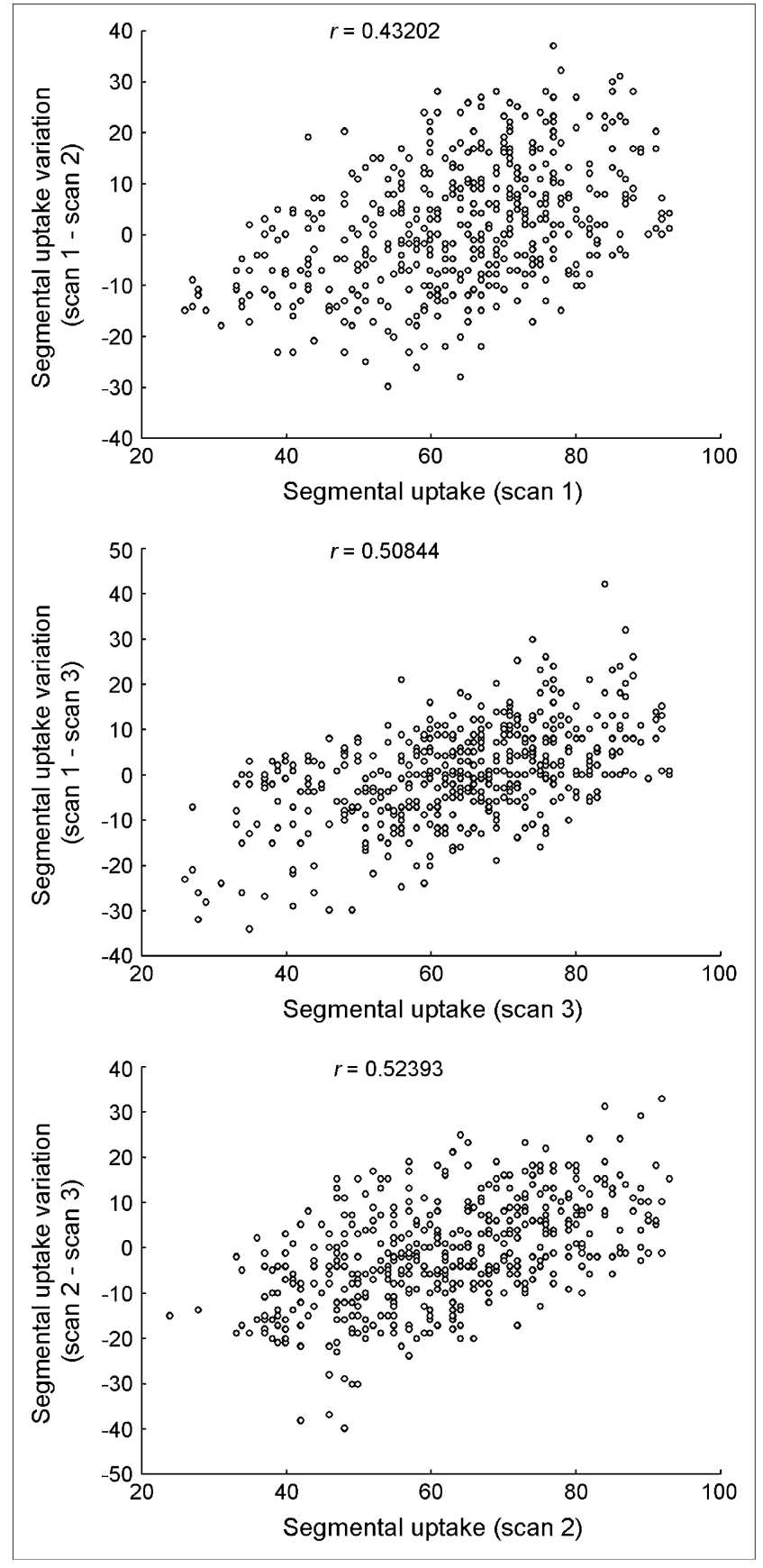

FIGURE 3. Correlation between segmental uptake at first test and segmental uptake variations between subsequent tests.

we did not observe any correlation between the heterogeneity of myocardial ${ }^{18} \mathrm{~F}-\mathrm{FDG}$ distribution and serum glucose level, the time between injection and imaging, or the global ${ }^{18} \mathrm{~F}-\mathrm{FDG}$ accumulation in the myocardium.

Alternatively, the nonuniform distribution of ${ }^{18} \mathrm{~F}-\mathrm{FDG}$ uptake in myocardial regions exposed to the same metabolic and hormonal signals might be attributed to technical and physiologic factors. These include technical artifacts such as partial-volume effect due to variable thicknesses of myocardial walls or real differences in glucose uptake related to
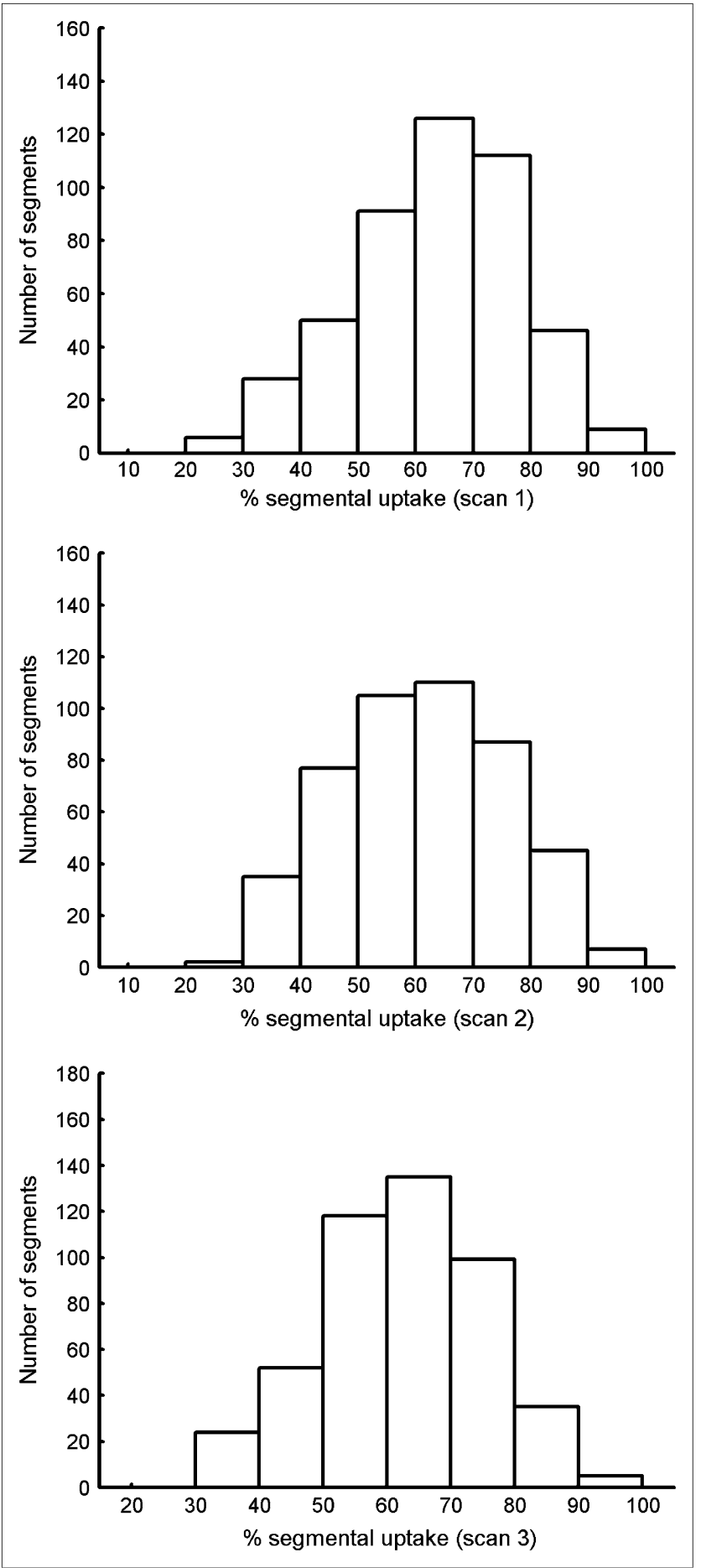

FIGURE 4. Spatial heterogeneity of regional ${ }^{18} \mathrm{~F}-\mathrm{FDG}$ uptake in LV myocardium. Frequency distribution of percent maximum ${ }^{18} \mathrm{~F}-\mathrm{FDG}$ uptake indicates a large variability across LV myocardial segments, even excluding the apical region. This finding was observed in a similar fashion at all 3 scans.

possible variability in contractile workload or in myocardial perfusion. However, all of these explanations seem extremely unlikely as the study was performed in subjects without cardiac history and, primarily, because these disparities disappeared or changed in sign at subsequent examinations. 
Unfortunately, the descriptive and retrospective nature of the present study prevents a conclusive definition of the mechanisms underlying the heterogeneous distribution of ${ }^{18} \mathrm{~F}$-FDG uptake in the myocardium of noncardiac patients under fasting conditions. However, these data clearly document that when a segmental analysis is performed according to the conventional models used in clinical nuclear cardiology, the ${ }^{18}$ F-FDG uptake can be as low as to define a "necrotic" pattern (i.e., a regional uptake $<50 \%$ of maximum myocardial concentration) in a significant number of instances, despite the absence of any sign or symptom of present or previous heart disease and, more importantly, despite the evidence of a significant likelihood of reversibility of the defect at subsequent examinations. Obviously, it must be considered that diagnostic myocardial ${ }^{18} \mathrm{~F}-\mathrm{FDG}$ imaging is most frequently used after glucose load or during hyperinsulinemic, euglycemic clamp (4). It is well known that both maneuvers remarkably smooth ${ }^{18} \mathrm{~F}$-FDG myocardial uptake. However, Gropler et al. (7) reported that spatial heterogeneity in ${ }^{18}$ F-FDG uptake does not disappear after glucose load in healthy subjects. Accordingly, these findings confirm that ${ }^{18} \mathrm{~F}$-FDG distribution alone should be used cautiously in the search of fingerprints of either myocardial ischemia or viability.

\section{Temporal Heterogeneity of Myocardial ${ }^{18}$ F-FDG Uptake}

The present study documents a large temporal variability of tracer distribution throughout the LV myocardium. Because we excluded patients with evidence of variability in global heart metabolism a priori, the relative stability of myocardial ${ }^{18} \mathrm{~F}$-FDG uptake over time cannot be considered as an index of stability of global myocardial metabolic pattern. On the other hand, the variability over time of global cardiac ${ }^{18} \mathrm{~F}-\mathrm{FDG}$ uptake is explained by several factors that are well described and related to the metabolic status of the patient as well as to the hormonal milieu at any given moment. This topic has been the subject of several previous experimental studies, leading to conflicting results. Khandani et al. (9) reported that subjective visual definition of cardiac uptake did not significantly change over time in 47 patients with 4 or more PET scans. On the other hand, de Groot et al. (10) showed that, in a subgroup of 25 patients who underwent at least 3 scans, visual grading of myocardial ${ }^{18} \mathrm{~F}-\mathrm{FDG}$ uptake did not change in only $36 \%$ of the cases.

Our data add to these previous observations by documenting a large temporal variability in regional metabolic pattern even in patients selected for a relative stability of glucose consumption in the whole heart. Accordingly, these data indicate that the spatial heterogeneity in glucose consumption was not caused by differences in myocardial metabolic pattern persisting over time and peculiar to the "geographic" definition of the myocardial region. Rather, each myocardial segment changes its own propensity to glucose consumption independently from its location as well as the behavior of the adjacent or remote myocardium.

\section{Limitations}

The retrospective nature of this study prevents identification of any possible mechanism underlying the large variability of myocardial metabolism. Moreover, as myocardial perfusion was not assessed, the present data cannot directly rule out the occurrence of small necrotic areas in the LV myocardium of these patients. However, it also should be considered that a high prevalence of myocardial necrosis is extremely unlikely in a series of patients without any history of cardiac disease. Moreover, and even more important, the longitudinal monitoring of cardiac ${ }^{18}$ F-FDG uptake documented that this variability occurred between different regions at the same times as well as between different times in the same region. This complex and random behavior indicates that the metabolic pattern should not be attributed to specific myocardial damage, is not peculiar of any given myocardial region, and might vary over time in different signs from one region to the other.

Again, the study design implied the evaluation of cardiac ${ }^{18}$ F-FDG uptake not in true control subjects but, rather, in cancer patients. Therefore, one must be very cautious in extending these data to the physiologic control of myocardial metabolism. Nevertheless, the observation of temporal and spatial heterogeneity documents that each myocardium responds to environmental signals in different ways at different times and locations. Thus, these data indicate that the interaction between myocardium and systemic signals is not homogeneous.

The analysis of temporal and spatial heterogeneity of regional myocardial uptake is restricted to only 26 of 49 patients because the software used for the semiquantitative segmental analysis failed in the automatic recognition of endocardial and epicardial borders in at least 1 of the 3 PET scan sequences in 23 patients. This is explainable as the software was originally developed to quantitate myocardial perfusion SPECT using ${ }^{201} \mathrm{Tl}$ or ${ }^{99 \mathrm{~m}} \mathrm{Tc}$-sestamibi and not ${ }^{18}$ F-FDG.

Because of the limited number of subjects, the present study does not allow evaluation of the effect of chemotherapy on myocardial ${ }^{18}$ F-FDG uptake. A possible effect of this treatment can actually be assumed because of not only the direct action of drugs on the myocardial tissue but also the changes induced in the metabolic status of the whole body. Similarly, because of the retrospective nature, we cannot exclude the possibility that the differences in metabolic patterns between LV walls might have been emphasized by the intervening chemotherapy (16). However, this limitation does not hamper the major aim of this study, as even this hypothesis implicitly assumes that the myocardial response to systemic stimulations or drugs might vary from one region to another at the same time or in the same region at different time points.

As a final limitation, one must consider that the diagnostic nature of PET studies implied ${ }^{18} \mathrm{~F}-\mathrm{FDG}$ injection under fasting conditions. It is well known that to optimize myocardial ${ }^{18} \mathrm{~F}$-FDG imaging, insulin stimulation is almost 
always enhanced by means of a glucose load or hyperinsulinemic, euglycemic clamp. According to the study of Gropler et al. (7), it seems conceivable that the observed heterogeneity would have been smoothed in studies performed under nonfasting conditions. Unfortunately, due to its retrospective nature, the present study does not allow us to address this concern. However, these data still indicate the need for a cautious interpretation of increased regional ${ }^{18} \mathrm{~F}$-FDG uptake as a marker of ischemia if not associated with a previous documentation of hypoperfusion.

\section{CONCLUSION}

The present study documents that the myocardial metabolic pattern can vary in different regions at the same time as well as at different times in the same region. This variability is extremely large and can be observed even in patients selected for a relative stability of metabolic pattern in the whole heart. These data have 2 potential implications. On the one hand, they indicate that uptake defects at myocardial ${ }^{18} \mathrm{~F}-\mathrm{FDG}$ imaging should be cautiously attributed to the presence of scar tissue, unless they are associated with the evidence of a severe hypoperfusion. On the other hand, these findings also indicate that one must be very cautious in extending the interpretation of studies on myocardial metabolism obtained by superselective cardiac vein drainage, as the observed phenomena might only partially reflect the metabolic pattern of the whole myocardium. Along this line of thought, imaging methods might provide a more comprehensive approach to define cardiac metabolism over time in each region of the LV myocardium.

\section{REFERENCES}

1. Tillisch J, Brunken R, Marshall R, et al. Reversibility of cardiac wall-motion abnormalities predicted by positron tomography. N Engl J Med. 1986;314:884-888.
2. Sambuceti G, Giorgetti A, Corsiglia L, et al. Perfusion-contraction mismatch during inotropic stimulation in hibernating myocardium. J Nucl Med. 1998;39: 396-402.

3. Gerber BL, Ordoubadi FF, Wijns W, et al. Positron emission tomography using ${ }^{18} \mathrm{~F}$-fluoro-deoxyglucose and euglycaemic hyperinsulinaemic glucose clamp: optimal criteria for the prediction of recovery of post-ischaemic left ventricular dysfunction-results from the European Community Concerted Action Multicenter Study on use of ${ }^{18} \mathrm{~F}$-fluoro-deoxyglucose positron emission tomography for the detection of myocardial viability. Eur Heart J. 2001;22:1691-1701.

4. Knuuti J, Schelbert HR, Bax JJ. The need for standardisation of cardiac FDG PET imaging in the evaluation of myocardial viability in patients with chronic ischaemic left ventricular dysfunction. Eur J Nucl Med Mol Imaging. 2002;29: 1257-1266.

5. Bonow RO, Dilsizian V, Cuocolo A, Bacharach SL. Identification of viable myocardium in patients with chronic coronary artery disease and left ventricular dysfunction: comparison of thallium scintigraphy with reinjection and PET imaging with ${ }^{18} \mathrm{~F}$-fluorodeoxyglucose. Circulation. 1991;83:26-37.

6. Marzullo P, Parodi O, Sambuceti G, et al. Residual coronary reserve identifies segmental viability in patients with wall motion abnormalities. J Am Coll Cardiol. 1995;26:342-350.

7. Gropler RJ, Siegel BA, Lee KJ, et al. Nonuniformity in myocardial accumulation of fluorine-18-fluorodeoxyglucose in normal fasted humans. J Nucl Med. 1990; 31:1749-1756.

8. Fukuchi K, Otha H, Matsamura K, Ishida Y. Benign variation and incidental abnormalities of myocardial FDG uptake in the fasting state as encountered during routine oncology positron emission tomography studies. $\mathrm{Br} J$ Radiol. 2007;80:3-11.

9. Khandani AH, Isasi CR, Blaufox MD. Intra-individual variability of cardiac uptake on serial whole-body ${ }^{18}$ F-FDG PET. Nucl Med Commun. 2005;26:787-791.

10. de Groot M, Meeuwis APW, Kok PJM, Corstens FHM, Oyen WJG. Influence of blood glucose level, age and fasting period on non-pathological FDG uptake in heart and gut. Eur J Nucl Med Mol Imaging. 2005;32:98-101.

11. Germano G, Kavanagh PB, Waechter P, et al. A new algorithm for the quantitation of myocardial perfusion SPECT. I. Technical principles and reproducibility. J Nucl Med. 2000;41:712-719.

12. Hays WL. Statistics. 4th ed. Fort Worth, TX: Holt, Rinehart and Winston Inc; 1988.

13. Streiner DL, Norman GR. Health Measurement Scales. New York, NY: Oxford University Press; 1989.

14. Tamaki N, Yonekura Y, Kawamoto M, et al. Simple quantification of regional myocardial uptake of fluorine-18-deoxyglucose in the fasting condition. J Nucl Med. 1991;32:2152-2157.

15. Choi Y, Brunken RC, Hawkins A, et al. Factors affecting myocardial 2-[F18]fluoro-2-deoxy-D-glucose uptake in positron emission tomography studies of normal humans. Eur J Nucl Med. 1993;20:308-318.

16. Panjrath GS, Jain D. Monitoring chemotherapy-induced cardiotoxicity: role of cardiac nuclear imaging. J Nucl Cardiol. 2006;13:415-426. 